\title{
1 Localization of infection in neonatal rhesus macaques after oral viral challenge
}

3 Roslyn A. Taylor ${ }^{1}$, Michael D. McRaven¹, Ann M. Carias ${ }^{1}$, Meegan R. Anderson ${ }^{1}$, Edgar

4 Matias $^{1}$, Mariluz Araínga ${ }^{2}$, Edward J. Allen ${ }^{1}$, Kenneth A. Rogers ${ }^{2}$, Sandeep Gupta ${ }^{3,4}$, Viraj

5 Kulkarni ${ }^{3}$, Samir Lakhashe ${ }^{3,4}$ Ramon Lorenzo-Redondo ${ }^{1,5}$, Yanique Thomas ${ }^{1}$, Amanda

6 Strickland ${ }^{4}$, Francois J Villinger ${ }^{2}$, Ruth M. Ruprecht ${ }^{2,3,4}$, Thomas J. Hope ${ }^{*}$

$7 \quad{ }^{1}$ Department of Cell and Developmental Biology, Northwestern University Feinberg School of

8 Medicine, Chicago, Illinois, United States of America

$9{ }^{2}$ Department of Biology, New Iberia Research Center, University of Louisiana at Lafayette,

10 Lafayette, Louisiana, United States of America

$11{ }^{3}$ Department of Microbiology, Immunology, and Molecular Genetics, University of Texas Health

12 San Antonio, San Antonio, Texas, United States of America

$13{ }^{4}$ Disease Intervention and Prevention, Texas Biomedical Research Institute, San Antonio, Texas,

14 United States of America

$15{ }^{5}$ Center for Pathogen Genomics and Microbial Evolution, Northwestern University Institute for

16 Global Health, Chicago, Illinois, United States of America

$18 *$ thope@northwestern.edu

20 Abstract

21 While vertical transmission of human immunodeficiency virus (HIV) can occur in utero and

22 during delivery and through breastfeeding. We utilized Positron Emission Tomography (PET)

23 imaging coupled with fluorescent microscopy of ${ }^{64} \mathrm{Cu}-$ labeled photoactivatable-GFP-HIV (PA- 
24 GFP-BaL) to determine how HIV virions distribute and localize in neonatal rhesus macaques

25 two and four hours after oral viral challenge. Our results show that by four hours after oral viral

26 exposure, HIV virions localize to and penetrate the rectal mucosa. We also used a dual viral

27 challenge with a non-replicative viral vector and a replication competent SHIV-1157ipd3N4 to

28 examine viral transduction and dissemination at 96 hours. Our data show that while SHIV-

29 1157ipd3N4 infection can be found in the oral cavity and upper gastrointestinal (GI) tract, the

30 small and large intestine contained the largest number of infected cells. Moreover, we found that

31 T cells were the biggest population of infected immune cells. Thus, thanks to these novel

32 technologies, we are able to visualize and delineate of viral distribution and infection throughout

33 the entire neonatal GI tract during acute viral infection.

35 Author Summary

36 Approximately 1.8 million children are currently living with human immunodeficiency virus

37 (HIV). While mother-to-child HIV transmission can occur in utero and during delivery, it most

38 commonly occurs through breastfeeding, creating the need to understand how the virus moves

39 throughout the body and infects the infant once breast milk is consumed. Here, we used multiple

40 imaging techniques and PCR to determine how HIV distributes throughout the gastrointestinal

41 tract after oral viral exposure and in which tissues and cell types become acutely infected. We

42 found that HIV rapidly spreads throughout and penetrates the entire gastrointestinal tract as early

43 as four hours after exposure. We also found that the intestine contained the largest number of

44 infected cells at 96 hours and that most cells infected were T cells. Our study shows that these

45 imaging technologies allow for the examination of viral distribution and infection in a rhesus

46 macaque model. 


\section{Localization of infection in neonatal rhesus macaques after oral viral challenge}

\section{$50 \quad$ Introduction}

$51 \quad$ Mothers living with human immunodeficiency virus (HIV) and not on antiretroviral

52 therapy have up to a $40 \%$ chance of passing HIV to their children [1]. Despite findings that

53 suggest that exclusively breastfeeding infants can reduce HIV acquisition [2,3], breastfeeding

54 remains one of the main routes through which vertical HIV transmission occurs [1,4]. This most

55 likely occurs during the transition from breastfeeding to the introduction of solid foods [5] and

56 possibly through the premastication of food by people living with HIV [6]. Regardless of their

57 antiretroviral regimen, many women living with HIV need to breastfeed their infants due to

58 limited resources in developing countries where the overall benefit of breastfeeding outweighs

59 the risk for other life threatening infectious diseases, creating a great need to understand the

60 mechanism of mother-to-child HIV transmission through oral viral exposure.

61 Non-human primates (NHP) provide a model of and an opportunity to investigate oral

62 vertical HIV transmission in vivo. NHP models of oral HIV viral exposure results in rapid

63 systemic infection using various methods of viral delivery as it has previously been shown that

64 four-week-old neonatal rhesus macaques (RMs) have high viral blood titers after oral challenge

65 with SIVmac251, which inversely correlated to survival [7]. Likewise, additional studies have

66 proven similar; for example, direct application of SIVmac251 to tonsils and the check pouch

67 resulted in systemic viral infection within seven days and two weeks, respectively [8,9]. Another

68 study showed SIVmac251 DNA was concentrated in tissues of the head and neck, and systemic

69 viral dissemination occurred four days after exposure when delivered dropwise into the mouth 
70 [10]. Lastly, recent reports also show that SIVmac251 RNA can be found in the brain and lungs

7172 and 96 hours after challenge and SIV DNA is found throughout the gastrointestinal (GI) tract

7296 hours after oral challenge [11].

73 HIV infects and depletes CD4+ cells in mucosal tissues, resulting in a decrease of CD4+

74 T cells in the blood $[12,13]$. CD4+ T cells in neonates have higher rates of cell metabolism,

75 proliferation and "activated" cell phenotypes [14,15] making them prime candidates for viral

76 infection. Therefore, the rapid spread of viral infection in infants is most likely due to this

77 immature highly metabolic immune system [16,17]. To demonstrate this CD4+ T cell

78 vulnerability to acute viral infection, Amedee et al., illustrated the presence of SIV RNA in tonsil

79 and mesenteric lymph node T cells 72 hours after oral challenge [11]. Furthermore, another study

80 reported that bottle fed neonatal RMs resulted in a reduction in CD4+ counts in the blood during

81 systemic infection a week after viral challenge [18]. Furthermore, neonates have a higher number

82 of CD4+ T cells in various tissues compared to adults $[19,20]$, which could contribute to viral

83 spread. In adult RMs, recent studies have focused on identifying which CD4+ T helper (Th) cell

84 subsets are highly susceptible to HIV/SIV infection, with an emphasis on Th17 cells [21]. It has

85 been shown that Th17 cells are preferentially infected in adult RMs after acute viral exposure in

86 the female reproductive tract [22]. Additionally, Th17 cells have been shown to play an

87 important role in HIV infection in the adult human colon $[23,24]$. The potential role of Th17

88 cells has not yet been elucidated in neonates.

89 Despite the advances in understanding mother-to-child transmission, the exact sites of

90 viral distribution and entry in breastfed infants remains unknown. This study utilizes three

91 different viruses (or virus-like particles) delivered in a bottle-fed neonatal RM model [25] to

92 examine possible sites of viral entry and infection after acute oral viral exposure. First, whole 
93 body Positron Emission Tomography and Computerized Tomography (PET/CT) imaging [26,27]

94 was coupled with fluorescent microscopy of a photoactivatable-GFP HIV-BaL (PA-GFP-BaL)

95 [28-30] to determine the distribution and localization of individual HIV virions two and four

96 hours after viral challenge. Using PET/CT guided necropsy, we determined that HIV virions

97 distribute to and penetrate the mucosal epithelium throughout the entire gastrointestinal (GI)

98 tract, including the rectum, four hours after oral viral exposure. To study viral infection and viral

99 cell targets, subsequent experiments used a dual viral challenge system, utilizing a non-

100 replicative reporter virus [31] and a replicative SHIV-1157ipd3N4 [32]. Using our non-

101 replicative reporter virus, we found the tongue may be a main site of viral transduction.

102 Additionally, in congruence with previous findings [9-11], we found that the entire GI tract is

103 susceptible to SHIV-1157ipd3N4 infection 96 hours after oral challenge. Likewise, the small

104 intestine was identified as the tissue that held the biggest foci of infection by fluorescent

105 deconvolution microscopy, with T cells encompassing the largest infected population of cells.

106 These findings can provide mechanistic insight and increase our understanding of mother-to-

107 child transmission after viral exposure.

108

109 Results

110 PET/CT imaging illustrates that HIV virions distribute throughout the GI tract four hours

111 after oral viral exposure

112 The technologies of PET/CT imaging, photoactivatable HIV (PA-GFP-BaL), and

113 fluorescent deconvolution microscopy [28-30] were combined to determine where virus

114 distributes throughout the body after oral exposure. PET/CT imaging allows for the in vivo

115 tracking of virions over time after challenge through radiolabeling PA-GFP-BaL with a 
116 radioactive isotope of copper, ${ }^{64} \mathrm{Cu}(\mathbf{F i g} \mathbf{1 A})$. During tissue processing, tissues are cut into $1 \mathrm{~cm}$

117 pieces, frozen into blocks with optimal cutting temperature medium (OCT), and each block is

118 scanned to identify individual blocks with positive radioactivity, thereby increasing our chances

119 of finding HIV virions by microscopy (Fig 1B and 1C [30]). These experiments occurred in

120 pairs, with one two-hour animal and one four-hour animal for each experimental day. The same

121 viral stock was used in all four animals.

122 Two hours after oral exposure of PA-GFP-BaL- ${ }^{64} \mathrm{Cu}$, PET scans revealed radioactivity

123 throughout the GI tract up to the transverse colon in both two-hour animals. Four hours after oral

124 exposure, radioactivity was observed throughout the GI tract to the colon including the length of

125 the rectum (Fig 2A-D). In one of the four-hour animals, PET signal was not found past the

126 descending colon, which was most likely due to a bubble of trapped gas that was observed upon

127 colon resection (Fig 2C). PET imaging of individual tissue blocks confirmed our findings from

128 the whole-body PET imaging in all four animals (Fig 1B); despite the gas obstruction observed

129 in one animal, individual radioactive blocks up to and including the transverse colon were found

130 in both two-hour animals and throughout the length of the GI to the rectum in both four-hour

131 animals (Fig 2C).

133 HIV virions distribute throughout the entire length of the GI tract four hours after oral

134 viral exposure

135 Next, fluorescent deconvolution microscopy was performed to identify the total number

136 of individual HIV virions (non-penetrating and penetrating) in individual tissue blocks that had

137 strong radioactivity by PET imaging (Fig 1C). Penetrating virions were defined as being one

138 micron from the epithelial surface. Because virions were not found in every tissue of the GI tract 
139 in all animals, the GI tract was dichotomized into upper (esophagus and stomach) and lower

140 (small intestine and colon) sections. Although the distance to which HIV virions distributed after

141 oral challenge extended to the transverse colon, the majority of the virions were located in the

142 oral cavity (buccal, tongue, and tonsil; mean $=26.5$ virions, frequency $=0.97$ ) in animals

143 necropsied two hours post-challenge. We also observed significantly fewer numbers $($ FDR $<0.05)$

144 and a lower frequency of virions both in the upper (mean $=1.67$ virions, frequency $=0.50)$ and

145 lower $($ mean $=2.25$ virions, frequency $=0.22)$ GI tract compared to the oral cavity two hours

146 after viral exposure (Fig 2E, F). However, in the four-hour animals, fewer virions were found in

147 the oral cavity (mean $=0.75$ virions, frequency $=0.03$ ). The highest number of the virions found

148 in the four-hour animals was in the lower GI tract (mean $=7.83$ virions, frequency $=0.78$; Fig

149 2E, F). The same number and frequency of virions were found in the upper GI tract (mean=1.67

150 virions, frequency $=0.50)$ in the four-hour animals as the two-hour animals.

151 Similar results were found when specifically examining the number of virions that

152 penetrated into the mucosa (Fig 2G). In the two-hour animals, the largest number of penetrating

153 virions were found in the oral cavity (sum of virions in both two-hour animals: non-penetrating =

15426 virions, penetrating $=80$ virions) with statistically significant differences observed both

155 between this cavity and the upper (non-penetrating $=3$, penetrating $=7$ ) and lower GT tract

156 (non-penetrating $=9$, penetrating $=15 ; \mathrm{FDR}<0.05$ ). Likewise, in the four-hour animals, the

157 majority of penetrating virions were found in the lower GI tract (non-penetrating = 19 virions,

158 penetrating $=75$ virions), although among the very few virions found in the oral cavity, all of

159 them were penetrating virions (penetrating =3). In the upper GI tract, few virions were found at

160 four fours (non-penetrating $=1$, penetrating $=8$ ) There was no difference in penetration depth

161 between location of virion or time point after oral challenge (Fig $2 \mathbf{H})$ with the exception of the 
162 few virions found in the oral cavity at four hours that proportionally were also significantly

163 deeper penetrators. These data, combined, demonstrate the validity of PET/CT to identify areas

164 of virus accumulation along with illustrating that HIV virions can distribute throughout the entire

165 GI tract four hours after oral viral exposure in neonates.

167 Validating our dual challenge oral transmission model

While PET/CT and PA-GFP-BaL- ${ }^{64} \mathrm{Cu}$ experiments allow for the visualization of virion

169 distribution and penetration into mucosal tissues, these technologies do not allow for the

170 examination of viral infection. Therefore, to study viral infection, subsequent experiments orally

171 challenged neonatal RM with LICh, a non-replicative reporter virus [31], and a replicative

172 SHIV-1157ipd3N4 [32]. Our lab has previously shown that we can locate and identify cells that

173 were first transduced by the challenge inoculum through a viral vector, LICh [31]. Due to the

174 lack of accessory genes in LICh, the replication process gets halted after one round of viral

175 integration, thus resulting in viral transduction. In a proof-of-principle experiment, one animal

176 was orally challenged with $8 \mathrm{~mL}$ of LICh alone and then sacrificed at 96 hours after challenge to

177 validate whether LICh can be used to identify sites of viral transduction after oral challenge.

178 Tissues from the GI tract were extracted and analyzed by IVIS. We were able to identify

179 luciferase signal on IVIS in the esophagus, neck lymph nodes, and stomach of neonatal RMs at

18096 hours after oral exposure (Fig 3A).

\section{Identifying tissues susceptible to viral transduction and infection}

183 Since we were able to observe luminescence by IVIS after oral challenge, we proceeded

184 to use a dual viral challenge model for the remaining experiments. We have previously shown 
185 that LICh can be used as a guide to locate areas of replicative foci of infection, as LICh

186 disseminates throughout the body similar to replicative viruses [22,31]. Because LICh does not

187 replicate, an SIV containing an R5-tropic HIV Clade C env genes, SHIV-1157ipd3N4, was used

188 to study viral replication. Because LICh does not contain accessory genes, we could distinguish

189 SHIV-1157ipd3N4 infected cells from LICh transduced cells by examining replicative viral

190 infection by gag DNA and protein. To identify tissues that are initially susceptible to viral

191 transduction and infection, genomic DNA was extracted and performed nested PCR to target

192 mCherry and gag DNA. In a proof-of-principle experiment, one neonatal RM (RM13) was

193 challenged with four feedings of $2 \mathrm{~mL}$ of $\mathrm{LICh}$ and a repeated low dose of SHIV-1157ipd3N4

194 (see Experimental Methods) and necropsied shortly after two days of viral challenge to examine

195 early transmission events. Results from the low dose challenge and 53-hour time point showed

196 less transduction and infection, as predicted. At 53 hours post-oral challenge, we found mCherry

197 DNA in the top of the tongue (Table 1). Gag DNA was found in the neck lymph nodes, stomach,

198 and small intestine at 53 hours post-challenge.

\begin{tabular}{|c|c|c|c|c|c|c|c|c|c|c|c|}
\hline \multicolumn{2}{|c|}{ Table 1: Distribution of LICH transduction and SHIV-1157ipd3N4 DNA throughout the GI tract after oral challenge } \\
\hline Animal & Challenge & Tongue & Cheek & Soft Palate & Tonsil & $\begin{array}{c}\text { Neck } \\
\text { Lymph } \\
\text { Nodes }\end{array}$ & Esophagus & Stomach & $\begin{array}{c}\text { Small } \\
\text { Intestine }\end{array}$ & $\begin{array}{c}\text { Large } \\
\text { Intestine }\end{array}$ & Spleen \\
Liver \\
RM13
\end{tabular} $\begin{gathered}\begin{array}{c}\text { Low Dose } \\
53 \mathrm{hrs}\end{array} \\
\text { RM10 }\end{gathered}$

199 LICh viral vector mainly found in the tongue after oral challenge. SHIV-1157ipd3N4 viral dissemination found

200 throughout the GI tract after oral challenge. + indicates mCherry DNA found; \# indicates gag DNA found 
For the next set of experiments, eight neonatal RMs were orally challenged with a repeated high dose challenge of SHIV-1157ipd3N4 and one $8 \mathrm{~mL}$ bolus of LICh, which was

204 given during the last bottle feeding, and the time between challenge and necropsy was extended

205 to 96 hours prior to nested PCR and fluorescent microscopy being performed, increasing the

206 potential of finding infected cells and of understanding the mechanism of viral transduction and

207 infection in our bottle-feeding model. While luciferase activity was previously observed in the

208 proof-of-principle study, when this group of eight neonates were examined by IVIS, very little

209 luciferase activity was seen among all the animals; therefore, we performed nested PCR on all

210 processed tissues. Nested PCR revealed mCherry DNA in the tongue, stomach, and small

211 intestine in RM10 and in the tongue of RM17. At 96 hours, SHIV-1157ipd3N4 viral

212 dissemination was more widespread. Gag DNA was found in the cheek, tonsil, soft palate,

213 esophagus, mesentery, small intestine, large intestine, liver, and spleen of RM10. Gag DNA was

214 also in the cheek, tongue, tonsil, the transformation zone to the stomach from the esophagus,

215 small intestine, large intestine, spleen, and neck lymph nodes of RM17 (Table 1). When

216 performing nested PCR, large amounts of tissues are sectioned for DNA isolation. Therefore, to

217 minimize tissue sample depletion, future experiments primarily focused on fluorescent

218 microscopy to identify viral transduction instead of utilizing nested PCR to identify infected

219 tissues.

\section{LICh transduction identifies initial target cells after oral viral challenge}

Tissue sections were then examined by fluorescent deconvolution microscopy to identify

223 individual LICh transduced cells at 96 hours (Table 2, Fig. 3). After a careful and thorough

224 examination of all tissues, an $\mathrm{mCherry}^{+}$cell was found in the tongue of RM22 (Fig. 3B). 
225 Spectral imaging confirmed that the emission spectrum on this cell matched the known emission

226 peak of mCherry, 610nm, (Fig. 3C), confirming that this cell in the tongue of RM22 was LICh

227 transduced from the challenge inoculum. Additionally, the trachea of RM23 also contained an

228 mCherry $^{+}$cell and the stomach of RM25 had several mCherry ${ }^{+}$cells. All the cells were validated

229 as $\mathrm{mCherry}^{+}$on spectral imaging (Table 2). However, although we were able to detect and verify

230 mCherry + cells in a few animals, these cells proved difficult to widely identify due to the high

231 autofluorescent background in the neonatal tissues. Therefore, tissue sections that contained

232 luciferase activity by IVIS were also stained with antibodies for luciferase. Unfortunately,

233 despite tissues showing luciferase activity by IVIS, positive luciferase signal was not detected in

234 any of the neonatal tissues (Fig. 3D). Although, we previously used a LICh viral vector in a

235 model of SIV infection in the female reproductive tract to identify sites of viral transduction [31],

236 these data suggest that in our oral viral challenge model, LICh technology may not be as

237 efficient. Therefore, defining SHIV-1157ipd3N4 viral infection, replication, and dissemination in 238 the oral and gut mucosa was prioritized.

\begin{tabular}{|c|c|c|c|}
\hline \multicolumn{4}{|l|}{$\begin{array}{l}\text { Table 2: Localization of LICh } \\
\text { transducedcells at } 96 \text { hours post oral } \\
\text { challenge by microscopy }\end{array}$} \\
\hline Animal & Tongue & Tonsil & Stomach \\
\hline RM10 & & & \\
\hline RM17 & & & \\
\hline RM22 & + & & \\
\hline RM23 & & + & \\
\hline RM25 & & & \\
\hline RM26 & & & \\
\hline RM27 & & & \\
\hline RM28 & & & \\
\hline
\end{tabular}


were validatedby spectral imaging. + indicates mCherry DNA found

Most infected cells are found in the small intestine after oral viral challenge

244 exposure, tissues were examined for evidence of SHIV-1157ipd3N4 infection using fluorescent

245 deconvolution microscopy (Table 3). Tissue sections from the oral cavity and GI tracts of each

246 animal were stained with antibodies directed toward SIV Gag (clone AG3) to identify SHIV-

247 1157ipd3N4 gag, as well as CD3 and CCR6 to phenotype infected immune cell subsets (Fig. 4,

248 Table 3). Five panels consisting of three 40x by five 40x images were taken for phenotype

249 analysis. As we have previously shown, these markers allow us to identify the target cells as

250 Th17, T cells, immature dendritic cells (iDCs), and other. In the tongues of neonatal RMs, we

251 found very small foci of infection; five of the eight animals examined had SHIV-1157ipd3N4

252 infected cells $(5.86 \pm 7.36$ cells, median $=4$, Fig. 4C). Similar to what was observed in the

253 tongue, the tonsils contained a small number of SHIV-1157ipd3N4 infected cells in six of the

254 animals $(13.5 \pm 19.93$ cells, median $=4)$, corroborating previous studies illustrating tonsils as a

255 site of potential viral entry after oral viral challenge $[8,10,18,33]$. The fewest number of infected

256 SHIV-1157ipd3N4 cells in terms of number of animals and quantity of cells were in the

257 esophagus $(5 \pm 12.55$ cells, median $=0.5)$. While SHIV-1157ipd3N4 infected cells were detected

258 in the tongue, tonsils, and esophagus, it was not a common event. In contrast, the stomach

259 contained larger foci of infection compared to those found in the oral cavity and esophagus; all

260 eight RMs had infected cells in the stomach $(43.63 \pm 60.82$ cells, median $=20)$.

Table 3: Localization of SHIV-1157ipd3N4 infected cells 96 hours after oral challenge by microscopy 


\begin{tabular}{|l|c|c|c|c|c|c|c|}
\hline Animal & Tongue & Tonsil & Esophagus & Stomach & Small Intestine & Large Intestine & Mesenteric Lymph Nodes \\
\hline RM10 & + & + & + & + & + & + & NA \\
\hline RM17 & & + & & + & + & + & + \\
\hline RM22 & & + & & + & + & + & + \\
\hline RM23 & + & + & + & + & + & + & NA \\
\hline RM25 & + & & & + & + & + & NA \\
\hline RM26 & + & + & + & + & + & + & NA \\
\hline RM27 & + & + & + & + & + & + & NA \\
\hline RM28 & & & & + & + & + & + \\
\hline
\end{tabular}

263 found by microscopy, NA indicates mesenteric lymph nodes were not collected from this animal

266 after intravenous infection [34]; therefore, we examined the small intestine, large intestine, and

267 their draining mesenteric lymph nodes for foci of SHIV-1157ipd3N4 infection to see if we could

268 identify similar after oral challenge. All eight RMs in the study had SHIV-1157ipd3N4 infected

269 cells throughout the small and large intestine. The small intestine contained the largest foci of

270 infection in all eight neonates $(367.1 \pm 250.8$ cells, median $=308$, Fig. 4A-B $)$. Overall, the large

271 intestine had the second largest foci of infection in the neonates $(159.4 \pm 88.39$, median $=176.5$,

272 infected cells, Fig 4.C, Fig. 5A). Unfortunately, we were only able to obtain the mesenteric

273 lymph nodes from three of the eight animals; however, we found SHIV-1157ipd3N4 infected

274 cells in the mesenteric lymph nodes of two of these three animals (Table 3). These data suggest

275 that viral replication can be found throughout the GI tract, all the way to the distal large

276 intestines and the corresponding mesenteric lymph nodes after oral viral challenge. 
The majority of SHIV-1157ipd3N4 infected cells throughout the gut are $T$ cells at 96 hours

279

280

281

282

283

284

285

286

287

288

289

290

291

292

293

294

295

296

297

298

299

300

\section{after oral viral challenge}

It has previously been shown that neonates have CD4+ T cells of a memory phenotype that proliferate at high rates in mucosal tissues, which may make these $\mathrm{T}$ cells the primary target of HIV/SIV infection [34]. Immature dendritic cells (DC) have been suggested to be initial targets and mediators of HIV/SIV [35-37] infection. Our lab has previously shown that Th17 cells are preferentially infected after vaginal SIV challenge [22]. Taking all these results together, we stained our tissue sections for T cell marker CD3 and the chemokine receptor CCR6 to investigate which cell types are infected after oral challenge. Infected cells were phenotyped into the following groups: Th17 T cells $(\mathrm{CD} 3+\mathrm{CCR} 6+\mathrm{AG} 3+)$, other T cells $(\mathrm{CD} 3+\mathrm{AG} 3+)$, Immature DC (CCR6+ AG3+), and other (AG3+). Due to the small number of SHIV-

1157ipd3N4 infected cells found in the tongue, tonsil, and esophagus, our phenotyping analysis focused on the stomach, small intestine, and large intestine. In the stomach, the majority of infected cells were other T cell subsets when examining all eight neonates combined $(n=242$

cells, 68.95\%, Fig. 5B). Overall, Th17 T cells were the second largest infected population in the stomach ( $\mathrm{n}=78$ cells, 22.22\%). Individually, Th17 T cells made up the largest group of infected cells in three of the RMs (RM17, RM22, RM26), while other T cell subsets were the largest group of infected cells in 4 of the RMs (RM23, RM25, RM27, RM28, SFig 1). Other cell types made up $6.55 \%$ of total infected cells $(n=23$ cells) and immature DC were $2.28 \%$ of infected cells $(n=8$ cells $)$ in the stomach. One neonate, RM10, had very few infected cells $(n=3)$, however $33.33 \%$ of infected cells of Th17 cells, $(n=1$ cell) other T cells subsets ( $n=1$ cell), and immature DC ( $\mathrm{n}=1$ cell, SFig 1). The largest infected population of cells in the small intestine was other T cells in all eight neonates ( $n=2468$ cells, $76.74 \%$, Fig 5B, SFig 1). The second 
301 largest infected population found in the small intestine was Th17 T cells ( $\mathrm{n}=655$ cells, 20.37\%).

302 Similar to the stomach, few other cell types ( $n=86$ cells, $2.67 \%)$ and immature DC $(n=7$ cells,

$3030.22 \%$ ) were infected in the small intestine. Other T cell subsets were marginally infected with

304 the most in the large intestine ( $n=651$ cells, $51.06 \%)$. For example, the large intestine was the site

305 of the greatest infection in Th17 T cells ( $n=608$ cells, 47.69\%, Fig 5B, SFig 1); the majority of

306 infected cells in half of the neonates were Th17 T cells (RM10, RM17, RM26, RM27), while

307 other T cell subsets were in the remaining four (RM22, RM23, RM25, RM28). Very few

308 infected immature DC ( $\mathrm{n}=10$ cells, $0.78 \%)$ and other cell types ( $\mathrm{n}=6$ cells, $0.47 \%)$ were found in

309 the large intestine. Overall, our data suggest that T cell subsets that are not Th17 are the greatest

310 target of infection in our oral transition model, unlike our previous findings after vaginal

311 challenge [22]. Among all of the animals and tissue types analyzed, very few immature DC and

312 other cell types were infected.

\section{Discussion}

315 Previous studies using intravenous inoculation of infant rhesus macaques have provided

316 great insights into the susceptibility of neonatal RMs to SIV infection [38,39] and CD4+

317 depletion in the gut after challenge $[14,15,34]$. However, breastfeeding remains the most

318 prevalent route for vertical HIV transmission worldwide [1]. This creates a great need to study

319 oral transmission in neonatal animal models. Here we show that, in a bottle-fed model, HIV

320 virions rapidly distribute throughout the GI tract and intact particles found as far as the rectum

321 four hours after oral exposure (Figs 1 and 2). We also show that the greatest number of SHIV-

322 1157ipd3N4 infected cells is in the small intestine and that T cells are primarily infected 96

323 hours after viral exposure (Figs 4 and 5). 
To determine how HIV virions distribute throughout the body immediately after oral exposure, we radiolabeled a photoactivatable, fluorescently tagged HIV and performed in vivo

326 PET imaging coupled with deconvolution fluorescent microscopy (Figs 1 and 2). While these

327 PET/CT experiments are not studying viral infection, these technologies provide insight into how

328 HIV disseminates throughout the body, and where in the oral mucosa and GI tract the virus

329 enters the mucosa after oral viral exposure. We found that two hours after oral challenge, HIV

330 virions mainly localize in the oral cavity, but then further disseminate to the transverse colon.

331 Four hours after challenge, HIV virions are found throughout the small and large intestine; in

332 both four-hour animals, many virions were also found within the rectum. These experiments

333 revealed that HIV distributes throughout the GI tract rapidly after oral challenge. In a matter of

334 two hours, the majority of total virions and penetrating virions changed from the oral cavity to

335 the lower GI tract consistent with virion penetration of epithelial barriers happening in a wave

336 followed by lysis or turnover because the signal was lost at the two-hour timepoint (Fig 2). It has

337 been reported that the gastric juice of neonates has a relatively neutral $\mathrm{pH}$ compared to adults,

338 ranging mostly between 7.5 and 8.5 , which could explain how the virus was able to survive and

339 pass through the stomach of the neonatal RMs [40].

340 Previously, it has been suggested that the tonsils may be a portal of viral access to

341 susceptible immune cells after oral exposure $[8,10]$; however, these studies directly applied virus

342 to the tonsils. We found very few SHIV-1157ipd3N4 infected cells in these tissues 96 hours in

343 our bottle-fed model of oral viral exposure (Figs 4C and 5A). Although these data do not

344 discount the tonsils as a potential source of initial viral entry, it suggests that this area may not be

345 the primary or major portal of entry for initial infection and viral dissemination. It is notable that

346 the tonsils are more similar to lymph nodes than the mucosal environment of the small and large 
347 intestine. Importantly, most of the T cells in the tonsil do not express the mucosal homing

348 receptor CCR5, which is a required co-receptor which in combination with CD4 is required for

349 the virion to functionally fuse with the target cell membrane. The paucity of susceptible T cells

350 (CCR5+) in the tonsils is the likely reason that we found limited numbers of SHIV-1157ipd3N4

351 infected cells in the tonsil and oral cavity overall. In contrast, the gut contains a large number of

352 potential SHIV-1157ipd3N4 target cells with CCR5+, CD4+ T cells being the majority

353 population in the immune cell rich mucosal environment of the intestine. This makes the gut a

354 rich environment for the virus to replicate which is reflected in the presented data, especially the

355 small intestine (Figs 4 and 5). Considering our observations, our data suggests that the gut may

356 represent the major portal of viral transmission after oral exposure by breastfeeding. Consistent

357 with this possibility, the distribution of viral particles in a liquid, oral challenge rapidly reaches

358 the stomach and intestines where there will be ample opportunity for the virus to reach and

359 penetrate the luminal barrier of the gut. In contrast, the fluid in the inoculum will rapidly flow

360 over the surface of the oral cavity giving less of an opportunity to penetrate the epithelial barriers

361 of the oral cavity which is target cell poor environment. While we found the majority of SHIV-

362 1157ipd3N4 cells in the small and large intestine, it is important to note that we cannot

363 determine with our methods if these large foci of infection represent the initial sites of infection

364 at the portal of transmission or a location rich in target cells that can foster high levels of viral

365 replication after initial expansion and replication in the oral cavity. It seems the large number of

366 expanding, substantial infectious foci in the gut most likely initiated in the gut considering the

367 short four-day period between drinking virus containing fluid and necropsy. The efficient

368 movement of the virus containing fluid we documented throughout the alimentary canal four

369 hours after drinking and our ability to identify intact viral particles penetrating the intestinal 
370 mucosa likewise support a model where oral exposure of virus containing fluid can utilize the

371 intestine as a portal of transmission.

372 Another retrovirus known to be milk transmitted is the mouse mammary tumor virus

373 (MMTV) that disseminates through the GI tract and directly infects immune cells in the Peyer's

374 patches of the gut, suggesting that other breast milk-transmitted retroviruses may also have this

375 capability $[41,42]$. Although current models of MMTV acquisition suggest that this process

376 takes place through cell-associated virus (infected cells in milk) which could withstand the acidic

$377 \mathrm{pH}$ of stomach acid better than cell-free virus [43]. However, our observations clearly

378 demonstrate the viral particle can reach the intestinal compartment intact, without being cell

379 associated, agreeing with previous results from Baba et. al. which demonstrated that cell-free

380 SIV is transmitted orally in neonatal rhesus macaques [38]. Providing the challenge virus in

381 formula may have played a role in buffering the mucosal environment slowing virion lysis and

382 degradation. Therefore, our PET/CT and microscopy data, taken together with the knowledge

383 that the gut is a target rich environment for viral infection is consistent with a model where the

384 SHIV-1157ipd3N4 infected cells we found in the intestines originated from virus in the

385 challenge inoculum utilizing the gut as the primary portal of transmission. Future experiments

386 are required to determine the origin of the foci of infection we observed.

387 Our lab has previously shown that the reporter viral vector, LICh, allows us to locate

388 tissues and cells that were transduced by the challenge inoculum through bioluminescence,

389 nested PCR and fluorescent microscopy [22,31]. This technology provides us with the ability to

390 identify cell types that are vulnerable to becoming the first cell infected in different models of

391 HIV transmission. In our model of oral HIV transmission, we were able to use LICh to identify

392 sites of viral transduction through the presence of mCherry DNA by PCR (Table 1). However, 
393 locating mCherry+ cells by microscopy was arduous (Fig 3 and Table 2). It may be possible

394 that, while our viral vector works well in rectal and vaginal infection models, this technology is

395 not well suited for oral challenge. Visualizing LICh is optimal with a focal area of transduction

396 to produce a sufficient density of photons for efficient detection. Therefore, a target poor

397 environment like the tonsils may not be able to achieve sufficient photon flux for detection.

398 Likewise, the particles will be greatly diluted and at low concentration to transduce cells at a

399 density conducive to the detection of luciferase activity. Because of inefficient photons to guide

400 our efforts to identify pieces of tissue containing foci of transduction, we decided to forgo

401 searching for LICh transduction in subsequent animals (RM23, RM25, RM26, RM27, RM28)

402 and proceed to identify SHIV-1157ipd3N4 infected cells. The large replicative capacity of the

403 virus, and infectious clone engineered to contain NF-KB sites in the long terminal repeats [32],

404 facilitated our ability to identify pieces of tissue containing foci of viral replication random

405 screening.

406 Models of mother-to-child transmission that have used oral routes of inoculation utilized

407 either dripping virus directly onto the tonsils [9,10,18,44], slowly dripping cell-free virus

408 solution onto the back of the tongue [38] or bottle-feeding methods $[18,45]$. To mimic how

409 vertical transmission occurs in humans via breastfeeding, we mixed challenge inoculums into

410 formula, which was then bottle fed [25]. Currently, the exact dose of HIV in each exposure

411 during breastfeeding in humans is unknown [1]. It has been shown that human breast milk viral

412 load can range widely from a hundred copies per milliliter to millions of copies per milliliter.

413 These studies have also shown that a high viral load in breast milk correlates to HIV

414 transmission in human infants [46-48]. Our initial experiments were based on repeated low dose

415 viral challenges as other studies have previously reported [18,44]. We found SHIV-1157ipd3N4 
416 DNA (Table 1), however, viral dissemination was not as widespread as we predicted using the

417 repeated low challenge dosing. Therefore, to better understand the mechanisms of viral

418 dissemination after oral challenge, we increased the dose of virus in our repeated challenges to

419 super-physiological. From these changes in experimental design, along with an increase in time

420 point to day four post viral challenge, infected cells could be detected easily throughout the GI

$421 \operatorname{tract}($ Figs 4, 5, and S1 and Table 3).

$422 \quad$ Neonates have an immature innate and adaptive immune system that is highly

423 metabolically active compared to adults [17], providing an optimal environment for viral

424 replication which often results in a high viremia. We have previously observed that immature

425 DCs are efficient targets of SIV infection after vaginal challenge [22,31]. They can also play

426 roles in HIV/SIV transmission in other models [35-37]. However, immature DCs are not a

427 preferential target of the SHIV-1157ipd3N4 virus utilized in this neonatal model of oral viral

428 exposure. For example, it has previously been shown that neonates have more proliferating

$429 \mathrm{CD} 4+\mathrm{T}$ cells in the small intestine than adult macaques and that these proliferating $\mathrm{T}$ cells are

430 selectively infected after intravenous injection of SIVmac251 in neonates $[14,15]$. Similarly, our

431 data show that T cells, specifically non-Th17 T cells, were the largest subset of infected immune

432 cells in all tissue types analyzed after oral SHIV-1157ipd3N4 challenge (Figs 5 and S1).

433 However, it is important to note that we did not look further into what CD4+ T cell subset makes

434 up our "other T cell" population. This remains to be investigated in future experiments.

435 These data demonstrate that HIV virions distribute throughout and penetrate the entire GI

436 tract at a very rapid rate hours after viral exposure. These findings were only made through use

437 of our technologies of PET/CT coupled with a photoactivatable GFP-tagged HIV. Furthermore,

438 we also show that the entire GI tract is susceptible to viral infection after oral viral exposure. Our 
439 data indicate that the small intestine is the primary site for viral infection and that CD4+ T cells

440 are the primary target cells 96 hours after viral exposure. Taken together, our results provide

441 more insight to the mechanism behind acute viral infection in neonates after oral exposure.

443 Experimental Methods

\section{Ethics Statement}

445 PET/CT experiments were conducted at the New Iberia Research Center (NIRC) at the

446 University of Louisiana at Lafayette. Studies examining LICh transduction and SHIV-

447 1157ipd3N4 infection were conducted at the Southwest National Primate Research Center at the

448 Texas Biomedical Research Institute (Texas Biomed), in San Antonio, Texas All procedures

449 were approved by the Animal Care and Use Committees at Texas Biomed (IACUC: $1441 \mathrm{MM})$

450 and NIRC (IACUC: 2017-8791-002). All studies were performed in accordance with the

451 recommendations in the Guide for the Care and Use of Laboratory Animals.

\section{Virus production}

453 To generate PA-GFP-BaL, we co-transfected the R5-tropic, R9-BaL infectious molecular

454 clone construct with a plasmid expressing a photoactivatable GFP (PA-GFP) [49] fusion with

455 HIV VPR (PA-GFP-VPR) as previously described [28-30]. The replication competent virus

456 labeled with PA-GFP-VPR generated by polyethylenimine transfection of human 293T cells in

457 DMEM medium containing 10\% heat-inactivated fetal calf serum, $100 \mathrm{U} / \mathrm{ml}$ penicillin, $100 \mu \mathrm{g} / \mathrm{ml}$

458 streptomycin, and $2 \mathrm{mM}$ l-glutamine. After 24 to 48 hours, virus was harvested, filtered at 0.45

$459 \mu \mathrm{m}$ and stored at $-80^{\circ} \mathrm{C}$ [28]. Viral particles were concentrated and enriched by centrifugation

460 through a sucrose cushion. 
LICh reporter virus was produced as previously described [31]. Briefly, a SIV-based

462 pseudovirus vector system was generated from modifications of the SIV3 vector system [50].

463 The firefly luciferase gene is expressed through a poliovirus internal ribosome entry site (IRES)

464 [51]. Transcription of both luciferase and mCherry are driven from the constitutive immediate-

465 early CMV promoter and their expression is enhanced by WPRE for robust expression. LICh is

466 produced by transfecting 293T cells with four plasmids: LICh reporter genome, SIV3+

467 packaging vector, REV expression plasmid DM121, and JRFL envelope. Viral supernatants were

468 collected 48 hours post-transfection, purified through $0.45 \mu \mathrm{m}$ filters, concentrated over sucrose

469 cushions, and resuspended in PBS. Concentrated virus was stored at $-80^{\circ} \mathrm{C}$.

470 SHIV-1157ipd3N4 was generated as previously described [32]. Naïve RM peripheral

471 blood mononuclear cells (PBMCs) were stimulated with concanavalin-A, followed by infection

472 with SHIV-1157ipd3N4 that was harvested from 293T cells in the presence of human IL-2

$473(20 \mathrm{U} / \mathrm{mL})$ and TNF- $\alpha(10 \mathrm{ng} / \mathrm{mL})$. The PBMC-derived virus stock has a $\mathrm{p} 27$ concentration of

$474227 \mathrm{ng} / \mathrm{mL}$ and $4 \times 10^{6} \mathrm{TCID}_{50}$ per $\mathrm{mL}$ as titrated in TZM-bl cells.

\section{DOTA-labeling of virus}

476 HIV virus was DOTA-labeled as previously described [26,30]. PA-GFP-BaL was labeled

477 with a dodecane tetraacetic acid (DOTA) chelator, which allowed for attachment of ${ }^{64} \mathrm{Cu}$. Two

478 buffers were prepared using a chelating resin to remove all free copper: $0.1 \mathrm{M}$ sodium phosphate

479 buffer ( $\mathrm{pH} 7.3)$ and $0.1 \mathrm{M}$ ammonium acetate buffer ( $\mathrm{pH}$ 5.5). Chelex 100 Chelating Resin (5g,

480 BioRad, Hercules, California) was added to $100 \mathrm{ml}$ of each buffer, incubated with stirring for 1

481 hour at room temperature, and filtered at $0.22 \mu \mathrm{m}$ for sterilization. Concentrated virus was

482 resuspended in PBS and a 1:10 volume of 1 M sodium bicarbonate added. DOTA-NHS-ester

483 (Macrocyclics, Dallas, Texas) was dissolved in the $0.1 \mathrm{M}$ sodium phosphate buffer. The two 
484 solutions were combined (0.3mg DOTA-NHS-ester per 500ng of virus, as detected by p24

485 assay), and incubated on a rocker in the dark at room temperature. After 30 minutes, the buffer

486 was exchanged for the $0.1 \mathrm{M}$ ammonium acetate using a Zeba column 40K (Thermo Fisher

487 Scientific, Waltham, Massachusetts), wash steps completed per manufacturer's protocol, and

488 virus (PA-GFP-BaL- $\left.{ }^{64} \mathrm{Cu}\right)$ collected and frozen for shipment to New Iberia Research Center

489 (NIRC) at the University of Louisiana at Lafayette.

$490 \quad{ }^{64} \mathrm{Cu}$ labeling of virus particles.

491 A solution of ${ }^{64} \mathrm{CuCl}_{2}$ (University of Wisconsin-Madison) was neutralized with Chelex-

492 treated $1 \mathrm{M} \mathrm{NH}_{4} \mathrm{OAc}$ (Sigma) to a $\mathrm{pH}$ of 5.5, and an aliquot ( $\left.185 \mathrm{MBq}\right)$ incubated with DOTA-

493 PA-GFP-BaL stock at $37^{\circ} \mathrm{C}$ for one hour. The sample was purified with a Zeba desalting spin

494 column (30K MWCO, Thermo Fisher), eluted with PBS (Thermo Fisher), and labeling

495 efficiency was evaluated. Labeled virus (10-37MBq) was mixed with unlabeled virus

496 immediately prior to oral challenge.

497 Non-human Primate Studies: oral viral challenge

498 In total, 14 Indian-origin rhesus macaques (Macaca mulatta) were used. Four animals

499 that were used for PET/CT experiments received $2 \mathrm{~mL}$ of PA-GFP-BaL $(1,000 \mathrm{ng} / \mathrm{mL})+0.25 \mathrm{~mL}$

500 of PA-GFP-BaL- ${ }^{64} \mathrm{Cu}((1,000 \mathrm{ng} / \mathrm{mL})$ in $2 \mathrm{~mL}$ of Pedialyte for a total volume of a $4.25 \mathrm{~mL}$ feeding

501 (25). One animal was inoculated with a single dose (8mL) of LICh reporter virus and sacrificed

502 at 96 hours to generate proof-of-principle data (Fig. 3). One animal was inoculated with four

503 doses of $2 \mathrm{~mL}$ of LICh $+3.5 \mathrm{~mL}$ SHIV-1157ipd3N4 (8mL of LICh and 14mL of SHIV-

504 1157ipd3N4 in total) and sacrificed at 53 hours for a low dose, early time point challenge (Table

505 1). The remaining eight animals were challenged via eight feedings of 5mL SHIV-1157ipd3N4

506 (for a total of 40mL of SHIV115ipd3N4 over the course of the experiment) + one dose of $8 \mathrm{~mL}$ 
507 LICh, which was included in the final feeding (Supplemental Table 1). All animals were

508 inoculated with virus that was mixed with Pedialyte via oral bottle feeding. Animals were

509 humanely sacrificed with an overdose $(100 \mathrm{mg} / \mathrm{kg})$ of pentobarbital while under isoflurane

510 anesthesia (Euthasol, Virbac, Westlake, Texas) or telazol anesthesia. For all experimental

511 conditions, the oral mucosa, entire gastrointestinal tract, spleen, liver, trachea, lungs, and neck

512 lymph nodes were removed. For PET/CT experiments, tissues were cut into $1-\mathrm{cm}^{2}$ pieces and

513 frozen in optimal cutting temperature (OCT) media (Thermo Fisher Scientific). Once frozen

514 tissue was no longer radioactive, it was shipped on dry ice to the Hope Lab at Northwestern

515 University. For experiments examining LICh transduced and SHIV-1157ipd3N4 infected cells,

516 samples were stored in RPMI after necropsy, and shipped on ice overnight to Northwestern

517 University.

518 NHP studies: imaging (PET, CT, and IVIS)

519 Animals were sedated with intramuscularly with $10 \mathrm{mg} / \mathrm{kg}$ Telazol/ketamine (Zoeis,

520 Parsippany-Troy Hills, New Jersey) and 1-3\% isoflurane in 100\% oxygen for the following

521 scans. The animal's body was immobilized in dorsal recumbency in a vacuum-sealed veterinary

522 positioner, and body temperature maintained with a warm air blanket (3M Bair hugger Model

523505 warming unit, Saint Paul, Minnesota) and water-circulating heating pads. In addition,

524 respiration, movement, and mucosal coloration (PET Scanner) were visually assessed. PET/CT

525 scans were acquired using a Philips Gemini TF64 PET/CT scanner. The final CT image was

526 compiled from 250 to 300 slices, depending on animal size. PET-CT combined images were

527 analyzed using MIM software. Standard uptake values were measured using the volume regions

528 of interest (ROI) tool and compared and normalized across animals. All scans lasted 20 minutes.

529 Initial PET scans were obtained immediately following oral viral challenge. Second scans were 
530 performed one hour after oral challenge. PET scans also occurred at two and four hours for final

531 in vivo tracking of radiolabeled virus. CT scans were performed immediately following the last

532 PET scan. After sacrifice, PET images of whole tissues were taken. After tissue processing, each

533 block was scanned and compared to a standard control for PET intensity.

534 Tissues were soaked in $100 \mathrm{mM}$ d-Luciferin (Biosynth) for a minimum of 10 minutes and

535 placed in In Vivo Imaging System (IVIS) machine to examine luciferase activity (Figure 1B).

536 Tissues positive for luciferase were cut into $1 \times 1 \mathrm{~mm}^{2}$ pieces and frozen in optimal cutting

537 temperature (OCT) media for PCR and microscopy. For experiments with PA-GFP-BaL, four

538 animals received $0.5 \mathrm{~mL}{ }^{64} \mathrm{Cu}-\mathrm{PA}-\mathrm{GFP}-\mathrm{BaL}$ and unlabeled $1 \mathrm{~mL}$ of PA-GFP-BaL in Pedialyte.

539 Animals were humanely sacrificed with an overdose of a solution containing pentobarbital

$540(100 \mathrm{mg} / \mathrm{kg})$ while under isoflurane anesthesia. For all experimental conditions, the oral mucosa

541 and GI tract were removed and separated by individual tissues, mesenteric lymph nodes were

542 separated from the colon and stored as individual lymph nodes. All tissues were frozen in

543 optimal cutting temperature media (OCT) for microscopy.

544 Nested PCR

545 Genomic DNA for nested PCR was isolated from frozen tissue sections embedded in

546 OCT using the Qiagen DNeasy Blood and Tissue Kit as per the manufacturer's instructions. The

547 initial nested PCR reactions were performed with 250ng of DNA per reaction and DreamTaq

548 (Thermo Scientific). The second round of PCR was performed using $2 \mathrm{ul}$ of the first-round

549 reaction products. For detection of LICh transduction, PCR was performed to identify mCherry

550 DNA using the following primers: outside forward 5'-ACATGTGTTTAGTCGAGG-3', outside

551 reverse 5'-CAGTCAATCTTTCACAAATTTTGTAATCC -3', inside forward 5'-

552 CCGACTACTTGAAGCTGTCCTT-3', and inside reverse 5’- 
553 GTCTTGACCTCAGCGTCGTAGT-3'. For detection of SHIV-1157ipd3N4 infection, PCR was

554 performed to identify gag DNA using the following primers: outside forward 5'-

555 ATTAGCAGAAAGCCTGTTGGAG-3', outside reverse 5'-

556 AGAGTGTCCTTCTTTCCCACAA-3', inside forward 5'-

557 CATTCACGCAGAAGAGAAAGTG-3', inside reverse 5'-GGTATGGGGTTCTGTTGTCTGT-

558 3'. Each DNA sample was tested in 12 replicates. Sequences were confirmed by extracting

559 DNA bands using Qaigen QIAquick Gel Extraction Kit and analysis with the second-round

560 primers.

\section{Fluorescent microscopy and image analysis}

$562 \quad$ For all imaging, twelve-to-fifteen-micron tissue sections were cut and fixed with $3.7 \%$

563 formaldehyde in PIPES buffer for 10 minutes at room temperature. To study distributions and

564 localization of PA-GFP-BaL, nuclei were stained with Hoechst (1:25,000, ThermoFisher

565 Scientific). Coverslips were mounted with DakoCytomation mounting medium (Burlington,

566 Ontario, Canada) and sealed with nail polish. Twenty to twenty-two Z-stack images at 0.5 - $\mu \mathrm{m}$

567 steps were obtained by deconvolution microscopy on a DeltaVision inverted microscope (GE,

568 Boston, Massachusetts) at 100x. The 20-22 images were taken in an unbiased manner by

569 following the linear path of the luminal surface of the mucosa with the distance between each

570 image dictated by bleaching caused by photoactivation. Images were analyzed with softWoRx

571 software (Applied Precision, Issaquah, Washington). Number of total virions and penetrating

572 virions were counted. Virions were defined by existing in more than three $\mathrm{Z}$ planes and the post-

573 activation signal had to be at least three times higher than the pre-activation signal. Penetrating

574 virions were defined by being at least $1 \mu \mathrm{m}$ deep into the epithelium; all penetration depths were

575 measured in microns. 
Tissue sections used to investigate infected cells were then washed in PBS and tissue

577 sections were blocked in donkey serum (10\% normal donkey serum, $0.1 \%$ Triton-X-100, 0.01\%

$578 \mathrm{NaN}_{3}$ ) for 30 minutes at room temperature; blocking solution was used for staining buffer

579 throughout experiment. For mCherry detection, tissue sections were stained with primary

580 antibodies for CD3 (one drop of the prediluted antibody per $200 \mu$ l staining buffer, clone SP7,

581 Abcam) and HIV envelope (1:300 AG3, AIDS Reagent Repository) for two hours at room

582 temperature. Slides were washed with PBS and then stained with secondary antibodies donkey

583 anti-rabbit-AF488 and donkey anti-mouse-AF647 (1:1000, Jackson ImmunoResearch, West

584 Grove, Pennsylvania) for one hour at room temperature. Nuclei were stained with Hoechst

$585(1: 25,000)$ for 10 minutes at room temperature. Adjacent tissue sections were stained with a

586 primary rabbit anti-firefly luciferase (1:200, Abcam) for two hours at room temperature. Slides

587 were washed with PBS and then stained with secondary antibodies donkey anti-rabbit-AF488

588 (1:1000). Nuclei were stained for with Hoechst (1:25000). Coverslips were mounted with

589 DakoCytomation mounting medium and sealed with nail polish. Images were taken in $3 \times 5$ Z-

590 stack panels at $0.5 \mu \mathrm{m}$ steps for 30 steps by deconvolution microscopy on a DeltaVision inverted

591 microscope (GE) at 60x. Images were analyzed with softWoRx software (Applied Precision,

592 Issaquah, Washington). Upon finding cells that appeared to be mCherry+, location of the cells

593 was recorded, and sections were marked. Slides were then taken to an A1R-Spectral confocal

594 microscope (Nikon, Tokyo, Japan) to analyze the emission spectra of the previously found cells

595 compared to the known emission spectra of mCherry at 610nm. Images were analyzed with NIS

596 Elements-C software (Nikon).

597 To phenotype of SHIV-1157ipd3N4 infected cells, slides were stained with primary

598 antibodies for CD3 (one drop of the prediluted antibody per $200 \mu$ staining buffer, clone SP7, 
Abcam) and HIV envelope (1:300 AG3, AIDS Reagent Repository) for two hours at room

600 temperature. Slides were washed with PBS and then stained with secondary antibodies donkey

601 anti-rabbit-AF488 and donkey anti-mouse-AF594 (1:1000, Jackson ImmunoResearch) for one

602 hour at room temperature. Tissue sections were then washed and stained with an AF647-directly

603 conjugated antibody towards CCR6 (1:200, clone G034E3, BioLegend) at $37^{\circ} \mathrm{C}$ for one hour.

604 Nuclei were stained for with Hoechst (1:25000). Coverslips were mounted with DakoCytomation

605 mounting medium and sealed with nail polish. Image panels containing 30 sections in the $\mathrm{Z}$

606 plane at 0.5um steps were taken and deconvoluted with softWoRx software on a DeltaVision

607 inverted microscope. Five 40x images were taken for each sample. Each image consisted of a

608 stitched panel of three 40x images by $540 x$ images to include the epithelium. Infected cells were

609 counted, cell phenotypes were identified $\left(\mathrm{T}\right.$ cells $-\mathrm{CD}^{+}$, Th17 cells $-\mathrm{CD} 3^{+} \mathrm{CCR} 6^{+}$, immature

610 dendritic cells - CD3-, $\mathrm{CCR}^{+}$, and other - CD3-, CCR66-), and cell subsets were recorded as

611 parts of a whole (100\%).

\section{Statistical Analysis}

613 All statistical analyses were performed using $\mathrm{R}$ version 4.0.2. To perform group comparisons per

614 necropsy time, different mixed-effects models were fitted separately for the 2- and 4-hour

615 necropsy animals. For each dataset, we used the best fitting model depending on the nature of the

616 data analyzed. We always included animal as a random effect in the models. Virion counts per

617 necropsy time were modeled using a negative binomial generalized mixed model to test for

618 differences between anatomical locations, controlling for the number of images taken per animal

619 and tissue. For this dataset we also tested zero-inflated negative binomial models due to the high

620 number of zeros observed in some anatomical regions, but these models did not significantly

621 improve the performance of the negative binomial model. To test for differences in number of 
622 penetrating virions present in the different anatomical locations per necropsy time, we

623 transformed the penetrating depth data into a binary categorical variable by considering

624 penetrating virions as those deeper than one micron from the epithelial surface data, while

625 defining the rest as non-penetrating virions. We subsequently fitted a binomial generalized linear

626 mixed-effects model including the anatomical location as the predictor variable, controlling for

627 the number of images taken per tissue and animal. Finally, we tested for differences in

628 penetrating depth among virions considered as penetrating by the previous definition between the

629 different anatomical locations. We used a linear mixed effects model, also controlling for

630 number of images. We performed all possible contrasts within each of the models, adjusting the

631 false discovery rate for multiple comparisons using Benjamini-Hochberg Procedure. A false

632 discovery rate (FDR) significance cut-off was set at $\mathrm{FDR}<0.05$ for every comparison.

633 Acknowledgements: Thanks to the research and animal care teams at Texas Biomed and NIRC.

634 Thank you to Danijela Maric and Katarina Kotnik Halavaty for instruction on experimental

635 techniques.

\section{References}

637 1. Abel K. The rhesus macaque pediatric SIV infection model - a valuable tool in 638 understanding infant HIV-1 pathogenesis and for designing pediatric HIV-1 prevention strategies. 639 Current HIV research. 2009;7(1):2-11.

640 2. Iliff PJ, Piwoz EG, Tavengwa NV, Zunguza CD, Marinda ET, Nathoo KJ, et al. Early 641 exclusive breastfeeding reduces the risk of postnatal HIV-1 transmission and increases HIV-free 642 survival. AIDS (London, England). 2005;19(7):699-708. 
643 3. Kuhn L, Sinkala M, Kankasa C, Semrau K, Kasonde P, Scott N, et al. High uptake of

644 exclusive breastfeeding and reduced early post-natal HIV transmission. PloS one.

$645 \quad 2007 ; 2(12): \mathrm{e} 1363$.

646 4. Overbaugh J. Mother-infant HIV transmission: do maternal HIV-specific antibodies 647 protect the infant? PLoS pathogens. 2014;10(8):e1004283.

648 5. Gaur AH, Dominguez KL, Kalish ML, Rivera-Hernandez D, Donohoe M, Brooks JT, et 649 al. Practice of feeding premasticated food to infants: a potential risk factor for HIV transmission. 650 Pediatrics. 2009;124(2):658-66.

651 6. Ivy W, 3rd, Dominguez KL, Rakhmanina NY, Iuliano AD, Danner SP, Borkowf CB, et al. 652 Premastication as a route of pediatric HIV transmission: case-control and cross-sectional 653 investigations. Journal of acquired immune deficiency syndromes (1999). 2012;59(2):207-12.

654 7. Van Rompay KK, Greenier JL, Cole KS, Earl P, Moss B, Steckbeck JD, et al. 655 Immunization of newborn rhesus macaques with simian immunodeficiency virus (SIV) vaccines 656 prolongs survival after oral challenge with virulent SIVmac251. Journal of virology. $657 \quad 2003 ; 77(1): 179-90$.

658 8. Stahl-Hennig C, Steinman RM, Tenner-Racz K, Pope M, Stolte N, Matz-Rensing K, et al. 659 Rapid infection of oral mucosal-associated lymphoid tissue with simian immunodeficiency virus. 660 Science. 1999;285(5431):1261-5.

661 9. Milush JM, Stefano-Cole K, Schmidt K, Durudas A, Pandrea I, Sodora DL. Mucosal innate 662 immune response associated with a timely humoral immune response and slower disease 663 progression after oral transmission of simian immunodeficiency virus to rhesus macaques. Journal 664 of virology. 2007;81(12):6175-86. 
665 10. Milush JM, Kosub D, Marthas M, Schmidt K, Scott F, Wozniakowski A, et al. Rapid 666 dissemination of SIV following oral inoculation. AIDS (London, England). 2004;18(18):2371-80.

667 11. Amedee AM, Phillips B, Jensen K, Robichaux S, Lacour N, Burke M, et al. Early Sites of 668 Virus Replication After Oral SIVmac251 Infection of Infant Macaques: Implications for 669 Pathogenesis. AIDS research and human retroviruses. 2018;34(3):286-99.

670 12. Douek DC, Brenchley JM, Betts MR, Ambrozak DR, Hill BJ, Okamoto Y, et al. HIV 671 preferentially infects HIV-specific CD4+ T cells. Nature. 2002;417(6884):95-8.

672 13. Kou J, Kuang YQ. Mutations in chemokine receptors and AIDS. Progress in molecular 673 biology and translational science. 2019;161:113-24.

674 14. Wang X, Rasmussen T, Pahar B, Poonia B, Alvarez X, Lackner AA, et al. Massive 675 infection and loss of CD4+ T cells occurs in the intestinal tract of neonatal rhesus macaques in 676 acute SIV infection. Blood. 2007;109(3):1174-81.

677 15. Wang X, Xu H, Pahar B, Alvarez X, Green LC, Dufour J, et al. Simian immunodeficiency 678 virus selectively infects proliferating CD4+ $\mathrm{T}$ cells in neonatal rhesus macaques. Blood. $6792010 ; 116(20): 4168-74$.

680 16. Jaspan HB, Lawn SD, Safrit JT, Bekker LG. The maturing immune system: implications 681 for development and testing HIV-1 vaccines for children and adolescents. AIDS (London, 682 England). 2006;20(4):483-94.

683 17. Simon AK, Hollander GA, McMichael A. Evolution of the immune system in humans from 684 infancy to old age. Proc Biol Sci. 2015;282(1821):20143085.

685 18. Abel K, Pahar B, Van Rompay KK, Fritts L, Sin C, Schmidt K, et al. Rapid virus 686 dissemination in infant macaques after oral simian immunodeficiency virus exposure in the 687 presence of local innate immune responses. Journal of virology. 2006;80(13):6357-67. 
688 19. Thome JJ, Bickham KL, Ohmura Y, Kubota M, Matsuoka N, Gordon C, et al. Early-life 689 compartmentalization of human $\mathrm{T}$ cell differentiation and regulatory function in mucosal and 690 lymphoid tissues. Nature medicine. 2016;22(1):72-7.

691 20. Rudd BD. Neonatal T Cells: A Reinterpretation. Annual review of immunology. $692 \quad 2020 ; 38: 229-47$.

693 21. Bixler SL, Mattapallil JJ. Loss and dysregulation of Th17 cells during HIV infection. 694 Clinical \& developmental immunology. 2013;2013:852418.

695 22. Stieh DJ, Matias E, Xu H, Fought AJ, Blanchard JL, Marx PA, et al. Th17 Cells Are 696 Preferentially Infected Very Early after Vaginal Transmission of SIV in Macaques. Cell host \& 697 microbe. 2016;19(4):529-40.

698 23. Hartigan-O'Connor DJ, Abel K, McCune JM. Suppression of SIV-specific CD4+ T cells 699 by infant but not adult macaque regulatory T cells: implications for SIV disease progression. The 700 Journal of experimental medicine. 2007;204(11):2679-92.

701 24. D'Ettorre G, Borrazzo C, Pinacchio C, Santinelli L, Cavallari EN, Statzu M, et al. Increased

702 IL-17 and/or IFN-gamma producing T cell subsets in gut mucosa of long-term treated HIV-1-

703 infected women. AIDS (London, England). 2019.

704 25. Van Rompay KK, Abel K, Lawson JR, Singh RP, Schmidt KA, Evans T, et al. Attenuated 705 poxvirus-based simian immunodeficiency virus (SIV) vaccines given in infancy partially protect 706 infant and juvenile macaques against repeated oral challenge with virulent SIV. Journal of acquired 707 immune deficiency syndromes (1999). 2005;38(2):124-34.

708 26. Santangelo PJ, Rogers KA, Zurla C, Blanchard EL, Gumber S, Strait K, et al. Whole-body

709 immunoPET reveals active SIV dynamics in viremic and antiretroviral therapy-treated macaques.

$710 \quad$ Nat Methods. 2015;12(5):427-32. 
711 27. Xie H, Wang ZJ, Bao A, Goins B, Phillips WT. In vivo PET imaging and biodistribution

712 of radiolabeled gold nanoshells in rats with tumor xenografts. Int J Pharm. 2010;395(1-2):324-30.

713 28. Carias AM, McCoombe S, McRaven M, Anderson M, Galloway N, Vandergrift N, et al.

714 Defining the interaction of HIV-1 with the mucosal barriers of the female reproductive tract.

715 Journal of virology. 2013;87(21):11388-400.

716 29. McDonald D, Vodicka MA, Lucero G, Svitkina TM, Borisy GG, Emerman M, et al.

717 Visualization of the intracellular behavior of HIV in living cells. The Journal of cell biology.

$718 \quad 2002 ; 159(3): 441-52$.

719 30. Taylor RA, Xiao S, Carias AM, McRaven MD, Thakkar DN, Araínga M, et al. PET/CT

720 targeted tissue sampling reveals virus specific dIgA can alter the distribution and localization of

721 HIV after rectal exposure. PLoS pathogens. 2021;17(6):e1009632.

722 31. Stieh DJ, Maric D, Kelley ZL, Anderson MR, Hattaway HZ, Beilfuss BA, et al. Vaginal

723 challenge with an SIV-based dual reporter system reveals that infection can occur throughout the

724 upper and lower female reproductive tract. PLoS pathogens. 2014;10(10):e1004440.

725 32. Song RJ, Chenine AL, Rasmussen RA, Ruprecht CR, Mirshahidi S, Grisson RD, et al.

726 Molecularly cloned SHIV-1157ipd3N4: a highly replication- competent, mucosally transmissible

727 R5 simian-human immunodeficiency virus encoding HIV clade C Env. Journal of virology.

$728 \quad 2006 ; 80(17): 8729-38$.

729 33. Tenner-Racz K, Stahl Hennig C, Uberla K, Stoiber H, Ignatius R, Heeney J, et al. Early

730 protection against pathogenic virus infection at a mucosal challenge site after vaccination with

731 attenuated simian immunodeficiency virus. Proceedings of the National Academy of Sciences of

732 the United States of America. 2004;101(9):3017-22. 
733 34. Veazey RS, Lifson JD, Pandrea I, Purcell J, Piatak M, Jr., Lackner AA. Simian

734 immunodeficiency virus infection in neonatal macaques. Journal of virology. 2003;77(16):8783-

73592.

736 35. Holl V, Xu K, Peressin M, Lederle A, Biedma ME, Delaporte M, et al. Stimulation of HIV-

7371 replication in immature dendritic cells in contact with primary CD4 T or B lymphocytes. Journal

738 of virology. 2010;84(9):4172-82.

739 36. Hu J, Miller CJ, O'Doherty U, Marx PA, Pope M. The dendritic cell-T cell milieu of the

740 lymphoid tissue of the tonsil provides a locale in which SIV can reside and propagate at chronic

741 stages of infection. AIDS research and human retroviruses. 1999;15(14):1305-14.

742 37. Wiley RD, Gummuluru S. Immature dendritic cell-derived exosomes can mediate HIV-1

743 trans infection. Proceedings of the National Academy of Sciences of the United States of America.

$744 \quad 2006 ; 103(3): 738-43$.

745 38. Baba TW, Koch J, Mittler ES, Greene M, Wyand M, Penninck D, et al. Mucosal infection

746 of neonatal rhesus monkeys with cell-free SIV. AIDS research and human retroviruses.

$747 \quad 1994 ; 10(4): 351-7$.

748 39. Marthas ML, van Rompay KK, Otsyula M, Miller CJ, Canfield DR, Pedersen NC, et al.

749 Viral factors determine progression to AIDS in simian immunodeficiency virus-infected newborn

750 rhesus macaques. Journal of virology. 1995;69(7):4198-205.

751 40. Miclat NN, Hodgkinson R, Marx GF. Neonatal gastric pH. Anesth Analg. 1978;57(1):98-

752101.

753 41. Dudley JP, Golovkina TV, Ross SR. Lessons Learned from Mouse Mammary Tumor Virus

754 in Animal Models. Ilar j. 2016;57(1):12-23. 
755 42. Ross SR. Mouse mammary tumor virus molecular biology and oncogenesis. Viruses.

$756 \quad 2010 ; 2(9): 2000-12$.

757 43. Wood LF, Chahroudi A, Chen HL, Jaspan HB, Sodora DL. The oral mucosa immune

758 environment and oral transmission of HIV/SIV. Immunological reviews. 2013;254(1):34-53.

759 44. Durudas A, Chen HL, Gasper MA, Sundaravaradan V, Milush JM, Silvestri G, et al.

760 Differential innate immune responses to low or high dose oral SIV challenge in Rhesus macaques.

761 Current HIV research. 2011;9(5):276-88.

762 45. Van Rompay KK, Schmidt KA, Lawson JR, Singh R, Bischofberger N, Marthas ML.

763 Topical administration of low-dose tenofovir disoproxil fumarate to protect infant macaques

764 against multiple oral exposures of low doses of simian immunodeficiency virus. The Journal of

765 infectious diseases. 2002;186(10):1508-13.

766 46. Ndirangu J, Viljoen J, Bland RM, Danaviah S, Thorne C, Van de Perre P, et al. Cell-free

767 (RNA) and cell-associated (DNA) HIV-1 and postnatal transmission through breastfeeding. PloS

768 one. $2012 ; 7(12):$ e51493.

769 47. Rousseau CM, Nduati RW, Richardson BA, Steele MS, John-Stewart GC, Mbori-Ngacha

770 DA, et al. Longitudinal analysis of human immunodeficiency virus type 1 RNA in breast milk and

771 of its relationship to infant infection and maternal disease. The Journal of infectious diseases.

$772 \quad 2003 ; 187(5): 741-7$.

773 48. Willumsen JF, Filteau SM, Coutsoudis A, Newell ML, Rollins NC, Coovadia HM, et al.

774 Breastmilk RNA viral load in HIV-infected South African women: effects of subclinical mastitis

775 and infant feeding. AIDS (London, England). 2003;17(3):407-14.

776 49. Patterson GH, Lippincott-Schwartz J. A photoactivatable GFP for selective photolabeling

777 of proteins and cells. Science. 2002;297(5588):1873-7. 
778 50. Nègre D, Mangeot PE, Duisit G, Blanchard S, Vidalain PO, Leissner P, et al.

779 Characterization of novel safe lentiviral vectors derived from simian immunodeficiency virus

780 (SIVmac251) that efficiently transduce mature human dendritic cells. Gene Ther.

$781 \quad 2000 ; 7(19): 1613-23$.

782 51. Rabinovich BA, Ye Y, Etto T, Chen JQ, Levitsky HI, Overwijk WW, et al. Visualizing

783 fewer than 10 mouse $\mathrm{T}$ cells with an enhanced firefly luciferase in immunocompetent mouse

784 models of cancer. Proceedings of the National Academy of Sciences of the United States of

785 America. 2008;105(38):14342-6.

786 Fig 1: PET imaging follows distribution of PA-BaL- ${ }^{64} \mathrm{Cu}$ after oral viral challenge Four

787 animals were orally challenged with PA-GFP-BaL- ${ }^{64} \mathrm{Cu}$. Two animals were sacrificed two hours

788 post challenge and another two four hours post challenge, and the oral cavity and entire GI tract

789 removed in one piece. The tissues were cut into pieces, frozen, cryosectioned, and prepared for

790 fluorescent microscopy. A,B) Representative PET images of neonates after oral viral challenge

791 with PA-GFP-BaL- ${ }^{64} \mathrm{Cu}$. Scale in Standard Uptake Value (SUV) (A) Whole body PET images at

792 two and four hours post-oral challenge. B) PET image overlayed on photograph of 25 individual

793 tissue blocks from oral mucosa and GI tract four hours post-challenge. C) Representative

794 fluorescent microscopy image showing individual HIV virions (red puncta indicated by white

795 arrows) penetrating the tongue of an animal that received PA-BaL- ${ }^{64} \mathrm{Cu}$ at two hours post-

796 challenge. Green - pre-activation, Red - post-activation (virion), Blue - Hoechst

798 Fig 2: HIV virions distribute throughout and penetrate the mucosal epithelium of the

799 entire GI tract four hours post-oral challenge Whole GI tracts were excised and imaged by

800 PET at two- and four-hours following necropsy. PET images overlayed on photographs allow for 
801 visualization of radioactivity throughout the GI tract at two hours (A-B) and four hours (C-D)

802 post challenge. White dotted lines depict where the GI tracts were dichotomized into upper

803 (esophagus and stomach) and lower (small and large intestines) regions. Quantification of virions

804 and viral penetration in the oral cavity, upper GI tract, and lower GI tract mucosa two and four

805 hours after oral viral challenge. E) Total number of virions counted in images. Means of the

806 virion count per group shown on stacked bars in white numbers. F) Frequency of total virions

807 counted. G) Quantification of the number of penetrating virions. Circle area represents the

808 number of virions. H) Virion penetration depth in microns. Each virion depth was truncated to

809 integer numbers for representation purposes. Circle area represents the number of virions with

810 the same depth value. Blue: two hours, Red: four hours

811

812 Fig 3: Detection of LICh transduced cells after oral challenge Eight animals were orally

813 challenged with LICh and SHIV-1157ipd3N4 and sacrificed at 96 hours. Tissues were dissected,

814 analyzed by IVIS, frozen and tissue blocks with high luciferase activity were cryosectioned.

815 Representative fluorescent microscopy images. A) Images of neonatal tissue showing luciferase

816 activity on IVIS B) Image of LICh transduced cell (Cell 2) in the tongue of RM22. Cell 2

817 indicates a possible mCherry+ cell, Cell 1 indicates a neighboring cell with red background

818 signal. Red - mCherry, green - CD3, Blue - Hoechst. 40x panel, scale bar is $30 \mu \mathrm{m}$ C) Spectral

819 imaging confirms mCherry signal in Cell 2 by comparison to known mCherry spectral emission.

820 Green - Cell 1, Red - Cell 2, Blue - known mCherry emission D) Image of tongue showing no

821 luciferase staining or mCherry label, suggesting LICh may not be sufficient in determining first

822 cell infected in neonate oral challenge model. Red - mCherry, green - luciferase, Blue -

823 Hoechst. 40x panel, scale bar is $30 \mu \mathrm{m}$ 
825 Fig 4: The majority of SHIV-1157ipd3N4 infected cells are found in the small intestine 96

826 hours after oral challenge. Fluorescent microscopy and quantification of SHIV-1157ipd3N4

827 infected cells from eight animals that were sacrificed at 96 hours after dual viral oral challenge.

828 Tissues were dissected, analyzed by IVIS, frozen, and tissue blocks with high luciferase activity

829 were cryosectioned. Slides were stained for CD3, CCR6, AG3, and Hoechst. Representative

830 fluorescent microscopy images. A-B) Cryosections of the small intestine showing SHIV-

831 1157ipd3N4 infected cells A) 40X panels, scale bars 60 microns B) 100x panels, scale bars 20

832 microns C) Quantification of total number of SHIV-1157ipd3N4 infected cells by tissue type.

833 Each dot represents the total number of cells found in five 40x panels in one individual animal

Fig 5: The majority of SHIV-1157ipd3N4 infected cells are T cells at 96 hours after oral

836 challenge Quantification of SHIV-1157ipd3N4 infected cells found in the oral cavity and GI

837 tract of animals examined in Figures 4-5. Graphs depict the percentage of infected cell types

838 identified by fluorescent microscopy as parts of a whole. Total cell counts were taken in five 40x

839 panels in every animal. A) Total number of infected cells in all eight neonatal RMs in tongue,

840 tonsil, esophagus, stomach, small intestine, and large intestine. B) Total number of infected cell

841 phenotypes in all eight RMs in the stomach, small intestine, and large intestine. Infected cell

842 types were categorized as five cell types: T cells (CD3+), TH17 T cells (CD3+, CCR6+), other T

843 cells (CD3+, CCR6-), Immature DCs (CD3-, CCR6+), Other (CD3-, CCR6-).

844

845 S1 Fig: Phenotype of SHIV-1157ipd3N4 infected cells in neonatal RM after oral viral

846 exposure Quantification of SHIV-1157ipd3N4 infected cells found in the GI tract of each animal 
847 examined in Figures 4-5. Graphs depict the percentage of infected cell types as parts of a whole

848 in each individual animal identified by fluorescent microscopy. Infected cell types were

849 categorized as five cell types: T cells (CD3+), TH17 T cells (CD3+, CCR6+), other T cells

850 (CD3+, CCR6-), Immature DCs (CD3-, CCR6+), Other (CD3-, CCR6-). Total cell counts were

851 taken in five 40x panels in every animal.

852

853 Table 1: Distribution of LICh transduction and SHIV-1157ipd3N4 DNA throughout the GI

854 tract after oral challenge LICh viral vector mainly found in the tongue after oral challenge.

855 SHIV-1157ipd3N4 viral dissemination found throughout the GI tract after oral challenge. +

856 indicates mCherry DNA found; \# indicates gag DNA found

857 Table 2: Localization of LICh transduced cells at 96 hours post oral challenge by

858 microscopy LICh transduced cells found in the tongue, trachea, and stomach 96 hours after oral

859 challenge by microscopy. Cells were validated by spectral imaging. + indicates mCherry DNA

860 found

861 Table 3: Localization of SHIV-1157ipd3N4 infected cells 96 hours after oral challenge by

862 microscopy SHIV-1157ipd3N4 viral dissemination found throughout the GI tract after oral

863 challenge. + indicates AG3 + cells found by microscopy, NA indicates mesenteric lymph nodes

864 were not collected from this animal

865 S1 Table: Identification of neonatal rhesus macaques used in LICh and SHIV-1157ipd3N4

866 studies *Day of harvest, date of harvest, initials of scientists who grew virus listed 
C)

CCR6 Ag3r Nuclei

Ags Fig4

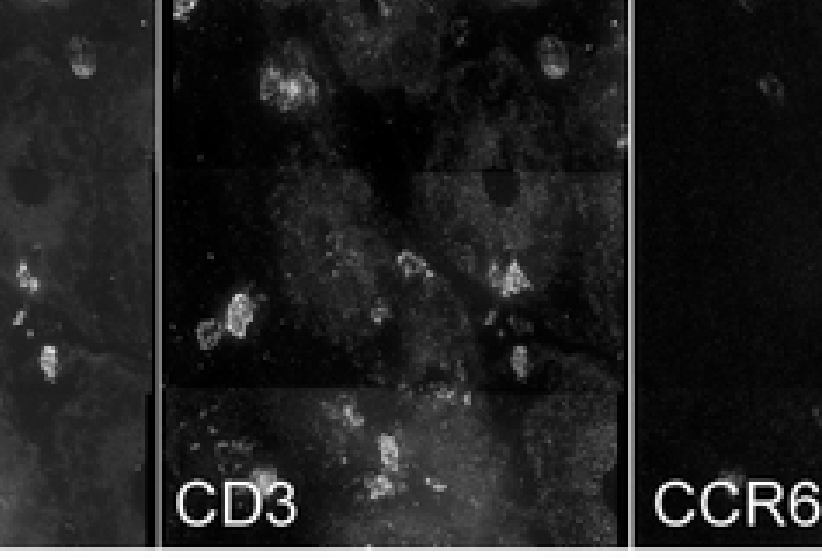
$a$

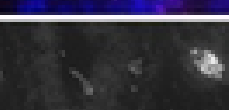
$\therefore$
CR6
Total infected cells $\quad$ RM10

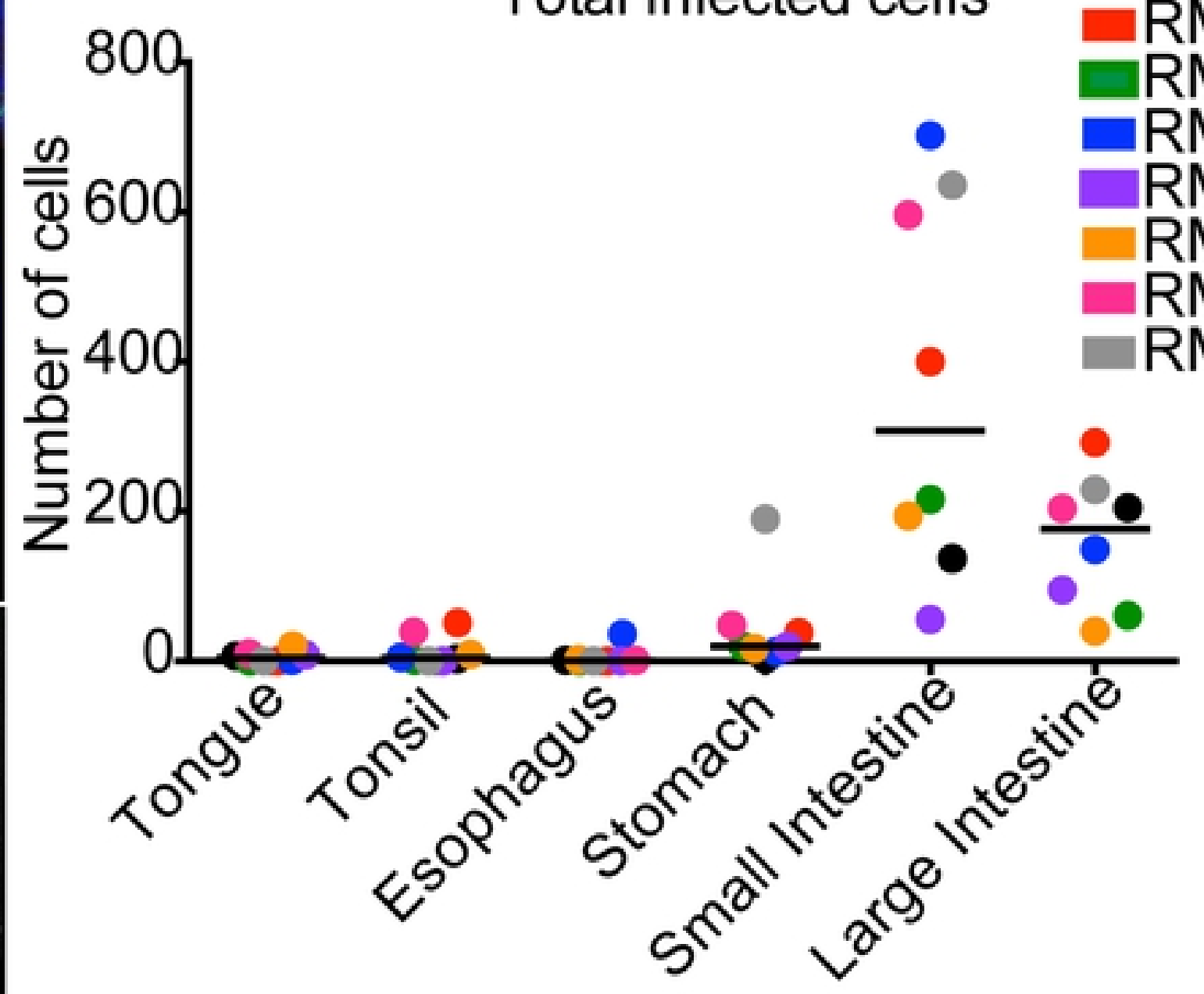
RM22 RM23 RM25 RM26 RM27 RM28 


\section{B) \\ Stomach \\ Large Intestine}

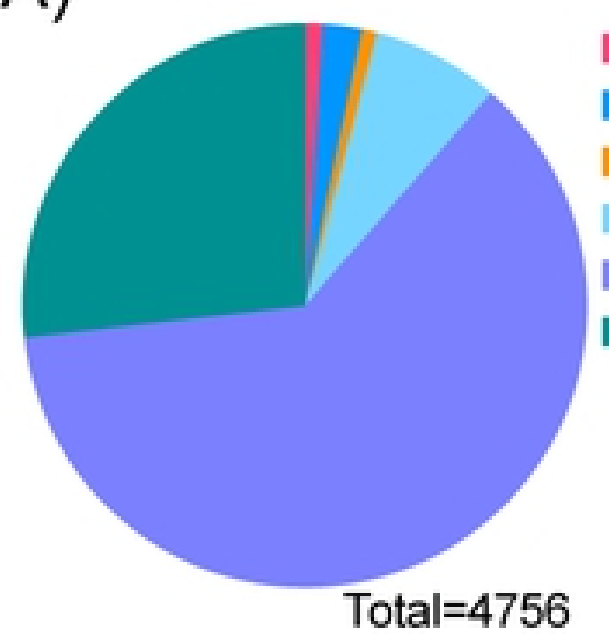

Tongue

Tonsil

Esophagus

Stomach

Small Intestine

Large Intestine 


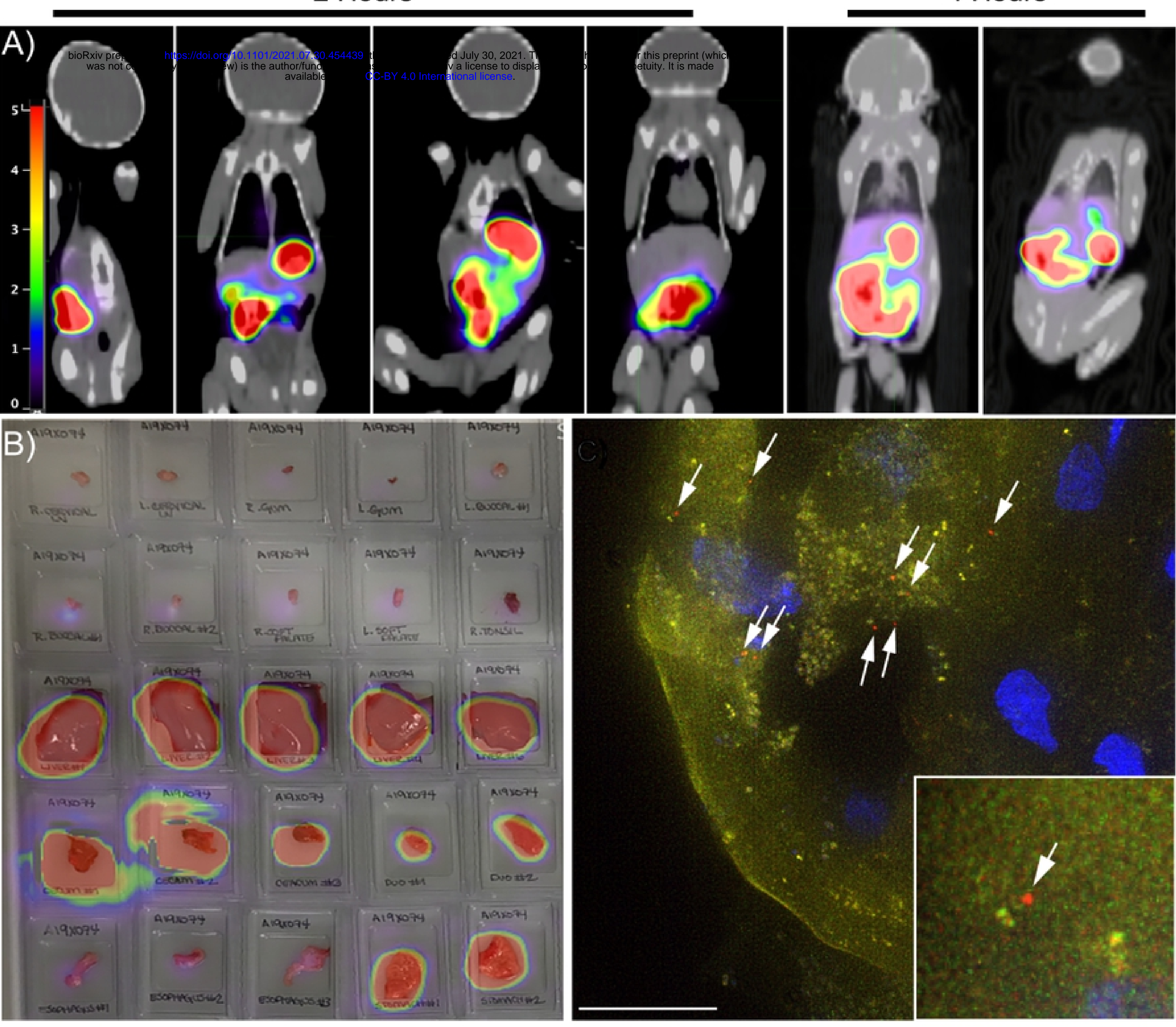

Fig1 
